\title{
Desempeño académico y experiencias de estudiantes universitarios mayas en Yucatán, México
}

\section{Performance academic and experiences of Mayan university students on Yucatan, Mexico}

\begin{abstract}
Rubi Surema Peniche Cetzal es docente de CONACYT-Universidad Autónoma de Aguascalientes (México) (rupeniche81@gmail.com) (http://orcid.org/0000-0003-0105-6471)
\end{abstract}

Cristóbal Crescencio Ramón Mac es docente de la Universidad Autónoma de Yucatán (México) (ccrmac@gmail.com) (http://orcid.org/0000-0003-3732-5717)

Recibido: 2017-07-25 / Revisado: 2017-08-28 / Aceptado: 2017-11-01 / Publicado: 2018-01-01

\section{Resumen}

Esta investigación fue realizada bajo una metodología cualitativa, empleando el estudio de caso colectivo. Se describen las experiencias académicas que han tenido los estudiantes universitarios mayas caracterizados con un desempeño académico sobresaliente, enfatizando en sus dificultades y las estrategias empleadas para sopesar tales situaciones. Los participantes fueron tres estudiantes universitarios mayas de una universidad pública en Yucatán, México. Se utilizó la entrevista semiestructurada y el diario de campo como técnicas de recolección. Al parecer, los tres casos se enfrentan al asunto económico como principal problema ante su formación profesional; la dificultad para emplear los equipos y recursos tecnológicos, la crítica a la imagen por parte de los demás, y la falta de apoyo y credibilidad por parte de los padres para formarse profesionalmente fueron otros obstáculos identificados. A pesar de ello, los estudiantes consideran que una correcta organización del tiempo, una adecuada comunicación con los profesores, una autogestión personal, así como el empleo frecuente de la tutoría institucional y los programas de becas a la excelencia, les permiten aminorar las dificultades y conseguir buenos resultados como universitarios. Mucho de los hallazgos fueron similares a la literatura que había sido reportada en años anteriores siendo lamentable observarlos en la actualidad.

Descriptores: Educación superior, desempeño académico, desigualdad social, pobreza, vulnerabilidad social,Yucatán, México.

\section{Abstract}

This research was performed under a qualitative methodology, using the collective case study. It describes the academic experiences that have been characterized with outstanding academic performance, college

Forma sugerida de citar: Peniche Cetzal, R. S. y Ramón Mac, C. C. (2018). Desempeño académico y experiencias de estudiantes universitarios mayas en Yucatán, México. Alteridad, 13(1), 120-131. https://doi.org/10.17163/alt.v13n1.2018.09. 
students emphasizing their difficulties and strategies used to weigh such situations. The participants were three Mayan University students of a public University in Yucatan, Mexico. The semi-structured interview and the field journal as data collection techniques was used. Apparently, all three cases is facing economic issue as a main problem in their vocational training; the difficulty of using the equipment and technological resources, criticism of the personal appearance by others, and the lack of support and credibility by parents for prepare professionally were identified like obstacles. Despite this,

\section{Introducción}

En América Latina creció el porcentaje de estudiantes entre 18 y 24 años de edad inscritos en educación superior de $21 \%$ en 2000 a $40 \%$ en 2010, en particular entre grupos de ingreso bajo y medio, en promedio, el 50\% más pobre de la población representaba el $16 \%$ de los alumnos de educación superior en 2000 y esta cifra creció alrededor de 25\% en 2013 (Banco Mundial, 2017), sin embargo y a pesar de los avances, en México se siguen observando amplias diferencias de cobertura en los contextos rural y urbano (Giorguli y Angoa, 2016; Solís, 2010) así como desigualdades de género para el acceso en varios estados del país, ya que las mujeres no han equiparado sus niveles de logro escolar respecto a los hombres, de acuerdo al censo de 2010, la brecha se hace más patente en el acceso a la universidad incluso en la capital cuando un $25 \%$ de hombres terminan su universidad completa respecto a un $18 \%$ de las mujeres que la concluyen (Brunet, 2016).

En la Educación Superior (ES) en México, sólo el 20\% de los jóvenes más pobres en el plano nacional acceden a la enseñanza superior, mientras aquellos con los ingresos más altos tienen una probabilidad cuatro veces mayor de cursar una carrera universitaria, desigualdad que evidencia la incapacidad del país para impedir que el origen de clase condicione el destino educativo, social y laboral de dicho sector (Peñaloza, 2011). En la educación se fundan los cimientos de la igualdad de oportunidades, siendo en México students considered that a proper organization of the time, a proper communication with professors, personal self-management, as well as the frequent use of the institutional mentoring and scholarship programs it excellence, allow them to lessen the difficulties and achieve good results as University students. Many of the findings were similar in the literature that had been reported in previous years and it is regrettable to still observe today. Keywords: Higher education, academic performance, social inequality, poverty, social vulnerability, Yucatan, Mexico.

esta desigualdad la que marca profunda y persistentemente las brechas sociales (Saraví, 2015). Las poblaciones vulnerables se ven afectadas por factores sociales como la pobreza que impiden ver a la escuela como un instrumento de mejora en la sociedad, la experiencia educativa se ve acotada por actividades como el trabajo infantil o adolescente que finalmente afectan el desempeño escolar; de igual manera, contribuye a las divisiones sociales mediante el acceso selectivo a las instituciones, según el origen socioeconómico de los alumnos como mencionó Tuirán (2011).

En México, el 10\% de la población pertenece a una de las 68 agrupaciones lingüísticas, que se encuentran localizadas principalmente en el centro, sur y sureste de México, geográficamente, los estados que pertenecen a esta zona, cuentan con índices bajo de desarrollo social y económico (Monroy, 2010) y estadísticamente las mujeres hablantes de lengua indígena alcanzan en promedio menos de 6 años de primaria (5.2 años), las brecha de género en los indígenas son muchos más grandes que las brechas étnicas. En particular, entre los hablantes de lengua indígena, los hombres tienen casi tres años en promedio más de asistencia a la escuela que las mujeres (Vázquez, 2016); del 27\% en jóvenes indígenas mayores de 15 años, únicamente el $1 \%$ de los indígenas que cursan la primaria acceden al nivel superior, de los cuales uno de cada cinco estudiantes egresa (Schmelkes, 2013) frente a los retos de acceso, permanencia y conclusión que implica realizar estudios universitarios (CONEVAL, 2011), de igual manera, que 
en el nivel educativo anterior no se han definido los parámetros mínimos sobre desempeños escolares y académicos (Zorrilla, 2010). Por otro lado, se ignora la cifra de estudiantes indígenas matriculados en educación superior, ya que las instituciones que no sean universidades indígenas e interculturales no reportan el origen étnico del estudiante (Didou, 2014).

El rezago educativo se vive en cada estudiante indígena que desea continuar con sus estudios y migra a las ciudades para tener acceso a una buena educación; la marcada brecha de calidad educativa debido a la inaccesibilidad geográfica, falta de servicios, oportunidades limitadas, así como la falta de recursos familiares arrastra marcadas deficiencias académicas (CONEVAL, 2011), con estas condiciones es casi inevitable que los resultados de aprendizaje sean muy pobres y por debajo de lo razonablemente esperable (Saraví, 2015), estos factores repercuten en el desempeño académico y se demuestra en los resultados desfavorables que presentan los estudiantes indígenas en pruebas estandarizadas como EXCALE, ubicándose en los niveles más bajos I y II, (INEE, 2010) frente a sus compañeros de ciudad que tienen trayectorias educativas diferentes, como lo indica Abiedi (2015), la pobreza limita el potencial de los estudiantes y eso impacta en el aprovechamiento académico. Además de la situación académica, el estudiante indígena tiene que enfrentarse a nuevos problemas que afectan su desempeño escolar por tener una cultura diferente a la de sus compañeros, el cambio de contexto de su lugar de origen a la ciudad que puede representar para la familia una pérdida importante, la incertidumbre por mantener los costos de traslado y manutención que genera el estudiar la educación superior y los factores discriminatorios que sufren quienes buscan y les atraen nuevas oportunidades de superación (Carnoy, Santibañez, Maldonado y Ordorika, 2002).

El acceso a la educación superior en México, es un privilegio que no todos los jóvenes mexicanos pueden presumir y quiénes logran ingresar hacen frente a factores que afectan su paso por este nivel educativo (Tuirán, 2011); factores como la homogenización social, la infraestructura pedagógica y la calidad educativa reproducen las desigualdades en el sistema educativo, las características sociales de los estudiantes en el este nivel educativo hacen de segmentación entre los estudiantes (Savarí, 2015). A pesar de ello, actualmente existen programas de apoyo destinados a jóvenes que cursan la educación superior para que continúen con sus estudios y disminuir las desventajas externas del nivel superior. A pesar de las oportunidades para continuar sus estudios, persisten problemas que afectan la finalización de los estudios, problemas, que contribuyen a reproducir la estratificación social (Blanco, 2014).

Específicamente, en el estado de Yucatán se han identificado ciertas causas sociales de rezago educativo a los estudiantes maya hablantes: la discriminación social principalmente por hablar su idioma nativo y los rasgos indígenas que poseen, así por la vestimenta tradicional; la familia de los estudiantes son otra causa de rezago, ya que al no contar con estudios, no ven la educación como un instrumento para tener mejores oportunidades y mejor calidad de vida; los medios de comunicación a los que acceden a través de la televisión, les parecen más atractivos que el asistir a la escuela; estas causas principalmente ocasionan que no puedan concluir la educación básica y llegar a la educación superior (Mijangos, 2009).

\section{Establecimiento del problema}

En México, al igual que en otros países de América Latina, es un privilegio el tener acceso a la educación superior y para la población indígena resulta ser más difícil por las pocas oportunidades de desarrollo, a pesar de estas contrariedades es esencial que los indígenas accedan a este nivel educativo (Schmelkes, 2003) que les permita tener una mejor calidad de vida y equidad social (Silas, 2010). 
Para acceder a las instituciones de educación superior, los aspirantes realizan un examen de admisión estandarizado y quienes logran incorporarse forman grupos heterogéneos de estudiantes y entre ellos se encuentran los estudiantes indígenas que han librado un obstáculo frente a la cultura dominante que hasta algunos años resultaban invisibles para las instituciones de educación superior (Carmona, 2013), si los estudiantes mestizos tienen diferencias académicas importantes, los estudiantes universitarios indígenas se encuentran en desventaja frente a los primeros que gozan al tener una madre lengua en español y una formación académica más consistente que se requiere para desarrollar estudios de nivel superior (Ortelli y Sartorello, 2011). Dubet (2005) identifica que una vez dentro de las instituciones de educación superior, los estudiantes universitarios se enfrentan a varios obstáculos en su experiencia escolar que tiene que ver con el tipo de organización universitaria, el contenido de los estudios, la finalidad de los estudios, las interacciones con sus compañeros $\mathrm{y}$ otros factores que influyen para permanecer o abandonar sus estudios.

Con base en lo anterior, Schmelkes (2003) menciona que los pocos estudiantes indígenas que logran ingresar a una institución de educación superior, consiguen desempeñarse exitosamente a pesar de las desventajas que tienen con respecto a sus compañeros que han tenido una mejor preparación. Savarí (2015) identifica dos dimensiones que viven los estudiantes por su paso por la escuela, la primera se refiere a la inclusión del individuo dentro de un grupo al que tiene que adaptarse y responder a la estructura establecida, el autor lo menciona como la experiencia del rol; la segunda dimensión se refiere a la experiencia particular en la trayectoria individual, es decir la propia experiencia biográfica.

Por ello, que surge el interés de conocer más sobre la vida escolar de los estudiantes universitarios mayas con desempeño académico sobresaliente, para saber cómo experimentan, significan e incorporan sus realidades en el nivel educativo que están estudiando, las situaciones y estrategias a las que se han enfrentado para permanecer en la universidad. Considerando que la población maya son jóvenes que provienen de familias maya hablante, hablan o entienden el idioma maya, en algunos casos provienen de comunidades rurales, tienen dificultades para acceder a la educación de nivel superior y que pocos son los que ingresan a la universidad, surge el planteamiento del problema de investigación con la siguiente pregunta:

¿Cuáles son las experiencias académicas que han tenido los estudiantes universitarios mayas caracterizados con un desempeño académico sobresaliente?

Ante la cuestión planteada, se presentan los siguientes objetivos que direccionan este reporte de investigación.

- Determinar los principales obstáculos que ha enfrentado el estudiante universitario maya con desempeño académico sobresaliente.

- Determinar las estrategias empleadas por los estudiantes universitarios mayas para obtener un desempeño académico sobresaliente.

Es importante comentar que el presente estudio formó parte de un proyecto macro de investigación que refirió a estudio de casos, pertenecientes a instituciones de educación superior ubicadas en el estado de Yucatán, México, y que se enfocó determinar las condiciones de desigualdad en ES en el Estado. Los casos de éxito identificados durante el estudio macro, dieron lugar al interés de este reporte con el fin de compartir los hallazgos, considerando que los sujetos de estudio fueron escasos, o que irónicamente convierte en relevante el estudio en su esencia.

\section{Fundamentación teórica}

La educación ha sido considerada como uno de los principales mecanismos de movilidad social y, por tanto, como uno de los instrumentos idóneos para combatir la desigualdad y mejorar la cali- 
dad de vida de los jóvenes mayas que están en la universidad (Gallart y Henríquez, 2006), sin embargo, en contraste se observan desigualdades para acceder en el sistema educativo mexicano que parecieran manifestar que el acceder al conocimiento es privilegio para los mejores posicionados, negando la posibilidad de desarrollo personal y social (OEI, 2010) y que de acuerdo a los enfoques sociológicos, pareciera que la escuela es únicamente un escenario para encaminar la reproducción de la estratificación (Zorrilla, 2010). Es interesante resaltar que los países más atrasados en el logro de la escolaridad universal para sus niños y adolescentes son precisamente los que tienen mayor proporción de población indígena (Ibarrola, 2014).

Desde mediados de la década de 1990, hubo un incrementó en la matrícula de educación superior de los países de América Latina, cabe mencionar que la tasa de atención fue desigual al interior de cada país en función a desigualdades económicas, culturales y entre los grupos de población (Ibarrola, 2014). Aun cuando los estudiantes mayas no tienen las mismas posibilidades de acceder a una institución de educación superior, hay algunos que logran ingresar a la universidad y permanecer hasta egresar con un desempeño académico sobresaliente pese a las dificultades sociales y económicas que enfrentan en su estancia en dicha institución (Rodríguez y Valdivieso, 2008). En una investigación realizada por los anteriores autores encontraron que el contexto social, económico y cultural en que vive un estudiante indígena tiene una influencia determinante en sus posibilidades de obtener un desempeño académico sobresaliente, que en la mayoría de las veces influyen positivamente en la autoestima del estudiante por mejorar cada día. Con base en lo anterior, los estudiantes mayas asimilan que unos ganan y otros pierden y que las recompensas, como las buenas calificaciones, las merecen sólo los que se han esforzado. Es por ello que aprenden la relación entre desempeño y gratificación, porque es una retribución, que premia el esfuerzo y la dedicación que el estu- diante obtuvo durante su estancia en la universidad (Ramírez, Devia y León, 2011).

El Estado le apuesta a la creación de programas que beneficien a los estudiantes mayas para permanecer en la educación superior y concluir una carrera universitaria. Un ejemplo es la creación del Programa Beca Manutención que ayuda al acceso y permanencia del estudiante en la universidad cuyo fin es brindar los recursos económicos para que el estudiante asista a la universidad y concluya sus estudios superiores; tiene como requisito que el alumno tenga un buen promedio y lo mantenga hasta finalizar sus estudios universitarios (Gallart y Henríquez, 2006). Cuando el estudiante de comunidades indígenas ingresa a la universidad cambia su comportamiento, su forma de vestir, actuar, su lenguaje, entre otros aspectos. Esto se debe a que al ingresar convive con personas de diferentes culturas y nivel socioeconómico diferente a la suya, sin embargo, algunos estudiantes, como los mayas, conservan su identidad cultural; en cambio, algunos jóvenes mayas adoptan nuevas ideologías de la cultura dominante para ser aceptados y que su estancia en la universidad sea más placentera, a lo que Edgardo Lander denomina como La colonialidad del saber (Schmelkes, 2003).

En su momento, Carnoy, Santibañez, Maldonado y Ordorika (2002) identificaron cinco obstáculos que sufren los estudiantes universitarios indígenas y que mantienen resistencia para permanecer en este nivel educativo. Estos obstáculos, tienen que ver con la distancia geográfica al no contar con instituciones de educación superior cerca de las comunidades ya que estas se encuentran en las capitales estatales, lo que obliga a emigrar; las barreras culturales, catalogada como una de las más importantes por ser una brecha de "choque migratorio" con problemas de adaptación y las propias barreras del lenguaje llegar a causar confusión en el sistema de comunicación y por lo tanto los pone en desventaja en el sistema educativo; las barreras económicas que impiden a las familias lidiar con el financiamiento de transporte, hospedaje, libros, alimentación, colegiaturas requeridos 
para estudiar la educación superior; la baja calidad educativa que recibieron en los niveles anteriores los pone en desventaja cuando realizan los exámenes de admisión para ingresar a la educación superior y lo factores de discriminación por su origen provoca problemas de interacción con los compañeros mestizos.

Algunos estudiantes universitarios, pertenecientes a zonas más urbanizadas, a pesar de contar con buenas condiciones materiales, un capital cultural adecuado y códigos culturales ampliados, no tienen desempeño académico sobresaliente en la escuela y, en el otro extremo, los estudiantes mayas universitarios que, a pesar de estudiar en las condiciones más desfavorables, obtienen un desempeño académico sobresaliente (Ramírez, Devia y León, 2011). Al respecto, Rodríguez y Valdivieso (2008), encontraron que los factores más importantes para que el estudiante universitario maya obtenga un desempeño académico sobresaliente no se deben al maestro, ni al currículo, ni al aparato escolar, sino a las condiciones del contexto familiar, aspecto que está fuera del ámbito de acción de las autoridades educativas. De forma adicional, identifican a la pobreza y al género como factores clave que mantienen a los estudiantes vulnerables, alejados de la escuela.

Frente a estos desafíos, los estudiantes indígenas con rendimiento académico sobresaliente instrumentan estrategias para permanecer y terminar exitosamente su carrera profesional, ya que refrendan el compromiso que adquieren con la familia al decidir estudiar fuera de su lugar de origen y valorar el esfuerzo de las familias que sacrifican la economía para que estudien (Chávez, 2008).

\section{Metodología}

El trabajo de investigación se realizó bajo el enfoque cualitativo, ya que ayudó a comprender la naturaleza de un problema, en este caso, las experiencias de estudiantes mayas con desempeño académico sobresaliente, para que los individuos puedan comprender su realidad. Se llevó a cabo un estudio de caso de tipo colectivo (Stake, 2005). Para este estudio los participantes fueron tres estudiantes universitarios mayas con desempeño académico sobresaliente de tres diferentes licenciaturas (enfermería, educación y contaduría) de una universidad pública ubicada en el oriente del estado de Yucatán, México; para la selección se establecieron características específicas: que los estudiantes estén inscritos en alguna de las tres licenciaturas antes mencionadas; que se encuentren en el rango de edad de 18 a 24 años; que tengan un promedio académico general en el rango de 90 a 100 y que sean estudiantes universitarios de origen maya.

Para obtener información para este estudio se utilizó la entrevista, debido a que busca entender el mundo desde la perspectiva del interesado y desmenuzar los significados de sus experiencias (Álvarez-Gayou, 2003). Otra técnica empleada fue el diario de campo (Flick, 2004), que se utilizó para obtener información actualizada y fue redactada por los sujetos de investigación durante cuatro meses, un reporte por semana, en el semestre agosto-diciembre de 2015. El tratamiento de los datos fue realizado mediante el análisis de contenido, que permitió el agrupamiento de las respuestas emitidas por los participantes en categorías específicas, para su posterior interpretación, como lo propone Creswell (2009). Para validar y verificar los datos obtenidos se utilizó una estrategia de triangulación metodológica: la entrevista, la revisión de la entrevista por parte de los entrevistados y el diario de campo, los cuales en conjunto complementan la recolección de los datos (Denzin citado por Flick, 2004).

\section{Resultados}

Considerando los objetivos del estudio, los resultados se reportan con base en cada uno de los tres casos, empleando pseudónimos para cuidar el anonimato y confidencialidad de los mismos. 


\section{Caso 1. Carlos}

\section{PRINCIPALES OBSTÁCULOS}

El obstáculo al que se ha enfrentado Carlos es al manejo y contacto con la tecnología, pues afecta su desarrollo en la universidad, más en el uso de la paquetería office al realizar las tareas escolares, lo que repercute en sus calificaciones; al parecer, cuando los estudiantes no están familiarizados con la tecnología se les dificulta adaptarse a los aparatos tecnológicos que se utiliza en su Institución de Educación Superior. Lo anterior se comprueba con el siguiente comentario:

Cuando entré a la escuela, yo sabía muy poquito usar las computadoras, y cuando me pedía tareas me ponía nervioso; creo que las hacía muy lento. Veía a mis compañeros y no les pasaba eso; el internet me costó mucho trabajo, el asunto de la búsquedas específicamente (Yucatán. Noviembre, 2015).

El asunto económico fue otra barrera para desarrollar sus estudios, ya que al carecer de recursos económicos no le permitió cubrir sus necesidades personales y académicas como estudiante universitario. De igual manera, se ha enfrentado al tiempo respecto del traslado desde su lugar de origen hacia la ciudad donde se encuentra la Universidad; al no contar con los recursos económicos para hospedarse en la ciudad donde se encuentra la universidad, tiene que viajar todos los días y, por ello, pierde tiempo que puede invertir en tareas académicas.

\section{Estrategias empleadas}

Carlos cree que una verdadera organización del tiempo puede ser la clave para obtener un nivel académico sobresaliente, debido a que aprovecha su tiempo seleccionando los espacios para estudiar, así como en terminar sus tareas académicas en tiempo y forma. Al parecer, este estudiante aprovecha las oportunidades que se le presentan en su vida universitaria está muy pendiente de las convocatoria que se publican, ya sea para concursar en eventos culturales (oratoria, maratones) o para evento académicos locales y nacionales (intercambios, veranos de investigación). Cree que es necesario tener trayectoria, relacionarse y hacer contactos en el campo profesional.

\section{Caso 2. Guadalupe}

\section{PRINCIPALES OBSTÁCUlos}

Al parecer, Guadalupe se ha enfrentado también al factor económico, pero referido más a tener que lidiar con asuntos de salud que con los escasos recursos monetarios que tiene no le permiten acudir a un médico especialista; ello le ha causado dificultades en la asistencia de clases y de tareas entregadas a destiempo. Aunado a ello, esta alumna tiene que trabajar en una papelería de su comunidad por lo que le cuesta tener tiempo suficiente para estudiar, pero es una necesidad imperante para obtener ingresos. Comentó:

...he tenido problema de gastritis y con el estrés se me empeora. A veces necesito comprar mis medicamentos pero no me alcanzan. Voy al servicio médico de mi universidad pero ahí solo me dan cosas remediales... el tiempo casi no me alcanza para hacer las tareas como quisiera, pero debo trabajar (Yucatán. Septiembre, 2015).

Por otro lado, Guadalupe considera como obstáculo el autoconcepto y autoimagen que se tenga, así como las creencias e influencia social externas que surgen por el hecho de ser de origen maya; lo anterior influye en la relación con los demás y de la inclusión al grupo. En ciertas ocasiones, algunos compañeros le comentan que habla con una acentuación maya muy marcada, y hasta en ocasiones le sugieren modificar su manera de vestir para actividades escolares o sociales.

\section{Estrategias empleadas}

Ante los obstáculos manifestados por la alumna, ella cree que una correcta organización de agenda 
le permite aprovechar los tiempos, incluso cuando tiene que trabajar de tiempo parcial. Cree que cuando se desea la superación, se busca cualquier espacio para cumplir las actividades y compromisos. De igual manera, mantener una comunicación estrecha con los profesores y expresar las dificultades es una adecuada estrategia para que los otros comprendan sus necesidades; aprender a negociar, hablar y trabajar en equipo le ha permitido avanzar a lo largo de los semestres.

Respecto de su identidad, esta universitaria trabaja todos los días con su autoconcepto; ha descubierto que posee un gran valor, pues sus compañeros le manifiestan su admiración y acuden frecuentemente por su ayuda ante las tareas. Lo anterior le permitido cierta fortaleza antes las críticas hacia su persona. Considera que no ha sido un camino fácil, y que en algún momento pensó en abandonar la carrera, pero ha descubierto mucho futuro viéndose como una profesional.

\section{Caso 3. Raquel}

\section{PRINCIPALES OBSTÁCULOS}

El principal obstáculo que ha enfrentado Raquel ha sido la falta de apoyo y credibilidad de sus padres y familia respecto de cursar una carrera profesional. Al parecer, la familia manifiesta que no es necesario estudiar tanto tiempo y no lograr un trabajo "bueno". A lo anterior se añade el rechazo hacia una carrera universitaria por el hecho de ser mujer, pues se ha de gastar tiempo y dinero en una formación que ni se ejercerá, al final la alumna seguro acabará casada y con hijos. Todo esto desgasta a Raquel, prefiriendo ignorar los comentarios y evitando compartir muchos de sus logros escolares.

Desde que inicié la carrera sabía que nadie me apoyaría, pero yo confío que valdrá la pena. Mi mamá piensa que no acabaré la carrera y que seguro estaré embarazada de algún fulano del pueblo. Yo le he dicho que no, pero no lo creen (Yucatán. Octubre, 2015).
Al igual que los anteriores casos, esta alumna se enfrenta todos los días a problemas económicos, pues debe trasladarse de un pueblo que se encuentra a 40 minutos del municipio además de los gastos que la propia carrera le exige. Lo más complicado es no contar con el apoyo económico de sus padres considerando la postura que ellos tienen y que ha sido descrito al inicio del caso.

\section{Estrategias empleadas}

Raquel considera que levantarse todos los días con una actitud propositiva es una buena estrategia para mantenerse de pie ante tanto obstáculo. Sabe que su condición de vulnerabilidad la hacen estar en desventaja ante sus compañeros pero cree firmemente que puede lograr la carrera. Intenta mantener una relación cercana con los profesores, pues ellos continuamente le dan consejos y le comparten materiales de lectura o productos que le proveen aprendizajes adicionales.

Aprovechando el programa institucional de Tutoría, esta estudiante confía en los beneficios de dicho sistema; cree que un adecuado acompañamiento le permite sopesar las dificultades diarias; en su caso, su tutora está muy pendiente de ella y continuamente revisan su expediente para establecer e implementar técnicas de estudio, en algunos casos se ha tenido que recurrir a la atención psicológica.

Adicionalmente, Raquel procura mantener la beca de manutención, a través de los programas del gobierno federal, que le proporciona un ingreso mensual; ello le permite cubrir sus necesidades básicas de la escuela.

\section{Discusión}

A pesar de las adversidades que enfrenta el estudiante maya en la universidad, algunos logran obtener un desempeño académico sobresaliente el cual es descrito como poseedor de conocimientos y habilidades con suficiencia y promoción que destaca del resto de su grupo de estudio (Erazo, 
2011). Siguiendo la misma línea del autor citado, para que un alumno tenga un desempeño académico ejemplar no basta con que salga bien, en un examen o en un curso, debe tener una conducta más o menos permanente, sistemática y recurrente, que le permita interiorizar las normas de la institución escolar, estudiar y "salir bien" de acuerdo con los parámetros de la escuela. Para ello debe tener la disciplina, los hábitos y el interés para dedicarse al estudio, por lo cual el alumno interioriza una actitud perseverante, debido a que sólo la capacidad intelectual no sería suficiente para sobresalir en el ámbito escolar.

Analizando los tres casos que conformaron esta investigación, se puede concluir que el asunto económico, escases de recursos monetarios, es un factor inevitable en el caminar de todo estudiante universitario, y mucho mayor en el caso de aquellos que vienen de comunidades rurales con el sueño de lograr una formación profesional. Muchos de ellos se enfrentan a la dificultad de adquirir materiales didácticos, alimentos durante su estancia en la jornada académica, pago del transporte desde su lugar de origen hasta la universidad, así como escasa o nula asistencia médica especializada; es entendible que al ser originario de una comunidad rural, la familia se dedica a actividades del campo y los ingresos son, en mayor parte, para cubrir las necesidades básicas del hogar; por ello, estos estudiantes difícilmente pueden obtener grandes apoyos monetarios por parte de sus padres o familiares. A pesar de lo anterior, en los tres casos se ha mostrado entereza para lograr mantenerse en su programa educativo, y con un desempeño sobresaliente. Aunado a lo anterior, Mijangos (2009) manifestó años atrás que la escasez de recursos económicos de las familias indígenas repercute directamente en los deseos de superación de jóvenes en el nivel superior. Esto queda claro, donde al parecer los programas gubernamentales no son suficientes en la cobertura o solvencia de esta necesidad. Parece necesario revisar qué es lo que se sigue haciendo, con un propósito claro de evaluación de políticas y programas sociales.
Respecto del limitado tiempo que tienen los estudiantes para realizar sus actividades académica, ya sea por trabajar de manera parcial o por el traslado de su comunidad al municipio principal, al parecer, una buena organización del tiempo y compromiso para ello permite obtener resultados óptimos, siendo similar a lo que Rodríguez y Valdivieso (2008) declararon. De igual manera, estos estudiantes consideran que ante el caso de sentirse agobiados ante su realidad, procuran mantener una actitud positiva y enfocada al futuro que los mantiene firmes antes los obstáculos.

A pesar que en la teoría reportada respecto que las mujeres indígenas se ven más limitadas para formarse profesionalmente según Brunet (2016), en la universidad a la que pertenecen los tres casos analizados en este estudio, dos fueron mujeres que mostraron desempeños académicos de gran reconocimiento en comparación de sus compañeros de aula no indígenas; ellas no solo tienen que enfrentarse a los típicas barreras de todo estudiante de comunidad rural, sino también a la falta de credibilidad de la familia y sociedad; al parecer, han roto esquemas y podrían convertirse en gestoras para la motivación de futuras generaciones, o agentes cambio. Relacionado a ello, parece ser que el profesor tiene un papel preponderante en el desempeño y crecimiento de los estudiantes, pues en los casos estudiados se mencionó que la relación y comunicación cercana con los maestros le permiten al alumno obtener conocimientos adicionales a los transmitidos en el aula, lo que indica que las interacciones con los formadores pueden motivar con mayor fuerza el interés de los jóvenes hacia la superación (Rué, 2009).

Vale pena considerar, que mucho de estas situaciones descritas como dificultades en la formación profesional, tiene que ver bastante con el proceso de adaptación que todo estudiantes enfrenta al iniciar los estudios superiores (Ferreyra, Avitabile, Botero, Haimovich, y Urzúa (2017), considerando que para algunos que se encuentran en condiciones de vulnerabilidad 
estas dificultades se potencializan por el simple hecho de venir de comunidades rurales o de formación académica previa con niveles deficientes, que incluyen no solo el poco manejo de las tecnologías, si no la misma interacción social.

\section{Conclusiones}

Es claro que este estudio no es generalizable a las poblaciones con condiciones similares, pero ante este fenómeno que es escasamente estudiado en el contexto, puede significar una contribución al trabajo científico y propuestas hacia la política educativa.

Ante lo anterior, las autoridades educativas deberían diseñar en las IES programas o proyectos que se enfoquen en los estudiantes universitarios indígenas, y en su caso con desempeño académico sobresaliente, para que desarrollen todas sus potencialidades y adquieran experiencias para su desarrollo profesional, buscando su motivación continua. Estos programas deben ser diseñados considerando las necesidades del contexto, con la participación de profesores y administrativos que vayan hacia la consecución de los mismos objetivos. Vale la pena mencionar, que al hablar de programas hacia una población específica no se está determinando la discriminación de la misma, lo que se pretende es que se atiendan ciertas necesidades que no son generalizables a toda la comunidad estudiantil por sus propias condiciones, incluso se esperaría que también pueda trabajarse en una cultura de inclusión dentro de la organización educativa. Parecería inherente la participación del sistema de educación media superior, por lo que debería invitársele al programa o proyecto que permita, desde sus funciones de formación, identificar estudiantes que se encuentren en las aulas y que cuenten con las características similares a los de este estudio; de esta manera, se podría anticipar casos que requieren de atención y promoción para concluir sus estudios de educación media y continuar así hacia la superior.
Respecto de los programas de apoyo o subsidio de estudiantes, es necesaria una revisión oportuna de la asignación y cantidad de recursos a aquellos alumnos que lo requieran, acompañado de un seguimiento efectivo de los mismos (Schmelkes, 2007). Es importante hacer a un lado, la concepción simple de que estos programas gubernamentales cumplen su función por el simple hecho de formar parte de políticas públicas, se requiere de campañas para una cultura de valorización que integra a la familia, sociedad y escuela. Trabajar hacia una equidad educativa en educación superior, es un terreno de gran amplitud (Köster, 2016); se debe comenzar con identificar la situación actual en el escenario educativo para determinar estrategias particulares que permitan una efectiva permanencia del alumnado.

Estudios como el descrito permiten mostrar el gran terreno que es necesario de atender a gran magnitud y con poblaciones mucho más amplias; es un espacio abierto a la comunidad científica para continuar el desarrollo de estudios que profundicen con otro tipo de diseños y técnicas para obtener información, desde perspectivas sociológicas y educativas.

\section{Referencias bibliográficas}

Abiedi, J. (2015). Student academic achievement and poverty. En W. Tierney, Rethinking education \& poverty (28-53). Baltimore: Johns Hopkins.

Álvarez-Gayou, J. (2003). ¿Cómo hacer investigación cualitativa?: Fundamentos y metodología. México: Paidós Educador.

Banco Mundial (Mayo, 2017). La educación superior se expande en América Latina y el Caribe, pero aún no desarrolla todo su potencial. México: BM. Recuperado de https://goo.gl/h39Tpd

Blanco, E. (2014). Interrupción de la asistencia escolar: desigualdad social, institucional y curso de vida. En E. Blanco, P. Solís, y H. Robles (Coords.), Caminos desiguales. Trayectorias educativas y laborales de los jóvenes en la Ciudad de México (39-70). México: COLMEX.

Brunet, N. (2016). Dejar la escuela en perspectiva longitudinal micro-macro: marcas biográ- 
ficas y contextuales. En M. Coubés, P. Solís, y M. Zavala (Coords.), Generaciones, cursos de vida y desigualdad social en México (339367). México: COLMEX.

Carmona, A. L. (2013). Las condiciones y estilos de vida de los estudiantes indígenas en la ciudad. Los universitarios en Xalapa. (Tesis de maestría). Xalapa: Universidad Veracruzana.

Carnoy, M., Santibañez, L., Maldonado, A., \& Ordorika, I. (2002). Barrera de entrada a la educación superior y a oportunidades profesionales para la población indígena mexicana. Revista Latinoamericana de Estudios Educativos, 32(3), 9-43.

Chávez, M. (2008). Ser indígena en la educación superior ¿desventajas reales o asignadas? Revista de la Educación Superior, 4(18), 31-55.

CONEVAL (2011). Informe de evaluación de la política de Desarrollo Social en México en materia de rezago educativo 2011. México: CONEVAL

Creswell, J. (2009). Research Design. Qualitative, Quantitative, and Mix Methods Approaches. Los Ángeles: SAGE.

Didou, S. (2014). Educación superior, afianzamiento de la identidad indígena y compromisos étnicos en México. Entre diversidades. Revista de Ciencias Sociales y Humanidades, 3, 17-49.

Erazo, O. (2011). Rendimiento académico, un fenómeno de múltiples relaciones y complejidades. Vanguardia psicológica, 2(2), 144-173. Recuperado de https://goo.gl/trCvCn

Flick, U. (2004). Introducción a la investigación cualitativa. Madrid: Morata.

Gallart, M., \& Henríquez, C. (2006). Indígenas y educación superior: algunas reflexiones. Universidades, 32, 27-37. Recuperado de https://goo.gl/YgvgRq

Giorguli, S., \& Angoa, M. (2016). Trayectorias migratorias y su interacción con los procesos educativos. En M. Coubés, P. Solís, y M. Zavala (Coords.), Generaciones, cursos de vida y desigualdad social en México (369-402). México: COLMEX.

Ferreyra, M., Avitabile, C., Botero, J., Haimovich, F., \& Urzúa, S. (2017). Momento decisivo: La educación superior en América Latina y el Caribe. Washington: Grupo del Banco Mundial.

Ibarrola, M. (2014). Una nueva educación para América Latina en el siglo XXI: desafíos, tensiones y dilemas. México: ANUIES.
INEE (2010). México en PISA 2009. México: INEE.

Köster, A. J. (2016). Educación asequible, accesible, aceptable y adaptable para los pueblos indígenas en México: Una revisión estadística. Alteridad. Revista de Educación, 11(1). https://goo.gl/7Bwn51

Mijangos, J. (2009). La lucha contra el rezago educativo. En el caso de los mayas de Yucatán. México: Unas letras.

Monroy, P. (en prensa) (2010, 28 de febrero). Educación en México, cada vez más pobre y desigual. Contralínea: Periodismo de investigación. Recuperado de: https://goo.gl/C1qgXC

OEI (2010). Metas educativas 2021: Desafíos y oportunidades. Informe sobre tendencias sociales en América Latina 2010. Buenos Aires: UNESCO-IIPE.UNESCO.

Ortelli, P., \& Sartorello, S. (2011). Jóvenes universitarios y conflicto cultural. Estudiantes indígenas y mestizos en San Cristóbal de las Casas, Chiapas. Perfiles educativos, 33, 115-128.

Peñaloza, P. (2011). El ocaso de la superstición punitiva. Revista el Cotidiano. 170, 39-48. Recuperado de https://goo.gl/yWFBhB

Ramírez, M., Devia, R., \& León, R. (2011). Pobreza y rendimiento escolar: estudio de caso de jóvenes con alto rendimiento. Educere, 52(15), 663-672. Recuperado de https://goo.gl/q2xC2v

Rodríguez, C., \& Valdivieso, A. (2008). El éxito escolar de alumnos en condiciones adversas. Estudios educativos, 38(1-2), 81-106. Recuperado de https://goo.gl/VkMBRY

Rué, J. (2009). El aprendizaje autónomo en educación superior. Madrid: Narcea.

Savarí, G. (2015). Juventudes fragmentadas. Socialización, clase y cultura en la construcción de la desigualdad. México: FLACSO, CIESAS.

Schmelkes, S. (noviembre, 2003). Educación superior intercultural el caso de México. Conferencia dictada en el Encuentro Internacional Intercambio de Experiencias Educativas: Vincular caminos a la educación superior. Recuperado de https://goo.gl/NHREY8

Schmelkes, S. (2007). El impacto del programa Oportunidades sobre la educación de la población indígena. Una exploración con base en estadísticas oficiales. En: $X$ Congreso Nacional de Investigación educativa, área 12: 
multiculturalismo y educación. Recuperado de https://goo.gl/F82cdC

Schmelkes, S. (1 de abril de 2013). Educación y pueblos indígenas: problemas de medición. Presentación en el panel sobre Vulnerabilidad educativa en el Seminario internacional Medición de grupos vulnerables. México: UNAM e INEGI.

Silas, J. (2010). Iniciativas para el acceso de la población indígenas a la educación superior en México. Pequeños pasos en un largo camino. Ponencia presentada en el $X$ Congreso Nacional de Investigación Educativa.

Solís, P. (2010). La desigualdad de oportunidades y las brechas de la escolaridad. En A. Arnaut, y S. Giorguli (Eds.), Los grandes problemas de México: educación (599-622). México: COLMEX.

Stake, R. (2005). Investigación con estudio de casos. 3a Ed. Madrid: Morata.

Tuirán, R. (2011). La educación superior en México: avances, rezagos y retos. Suplemento Campus Milenio. 27 de febrero.

Vázquez, G. (2016). Poblaciones indígenas urbanas en México. En M. Coubés, P. Solís, y Zavala, M. (Coords.), Generaciones, cursos de vida y desigualdad social en México (109-137). México: COLMEX.

Zorrilla, J. (2010). El bachillerato mexicano: un sistema académicamente precario. Causas y consecuencias. México: IISUE. 